Revista Digital Universitaria

Vol. 23, Núm. 1, enero-febrero 2022

\title{
Enfermería-Consulta: una plataforma digital que apoya el aprendizaje
}

\author{
Blanca Judith Lavoignet Acosta, Fabiola Cruz Núñez, \\ Guadalupe Santes Saavedra y Cynthia Lara González
}

\begin{abstract}
Resumen
De acuerdo con estimaciones emitidas por la Organización de las Naciones Unidas para la Educación, la Ciencia y la Cultura (UnESCO), más de mil 500 millones de estudiantes de 165 países no pueden asistir a los centros de enseñanza debido a la covid-19, esto ha obligado a la comunidad académica a explorar otras formas de enseñar y aprender, incluida la educación a distancia y en línea. Esta situación ha resultado difícil para estudiantes y docentes. Ante esta problemática, se creó e implementó un proyecto enfocado en un espacio digital como recurso didáctico, que brinda conocimientos a los estudiantes de enfermería, específicamente a los que cursan las experiencias educativas de Enfermería Farmacológica, Anatomía y Fisiología, y Práctica Profesional Comunitaria. Estos conocimientos están fundamentados en la ciencia, teoría y, a su vez, sirven para el desarrollo de habilidades-destrezas. El medio o herramienta utilizada es el Web log${ }^{1}$, cuya aplicación arrojó resultados muy satisfactorios. El objetivo de este artículo es presentar una herramienta que nos ha sido de gran utilidad en esta nueva normalidad. En conclusión, dicho proyecto también permite que el estudiante funja como el actor principal en su aprendizaje, al ser autónomo e independiente en la búsqueda de sus saberes.
\end{abstract}

Palabras clave: enfermería, plataforma digital, aprendizaje, blog, consulta.

\section{NURSING-CONSULTATION: A DIGITAL PLATFORM that SUPPORTS LEARNING}

\begin{abstract}
According to estimates issued by UNESCO, more than 1.5 billion students from 165 countries do not attend schools due to COVID-19, this has forced the academic community to explore other ways of teaching and learning, including distance and online education. This situation has been difficult for students and teachers. Faced with this problem, a project focused on a digital space as a didactic resource was created and implemented, which provides knowledge to nursing students, specifically those who are studying Pharmacological Nursing, Anatomy and Physiology, and Community Professional Practice. This knowledge is based on science, theory and, in turn, serves to develop skills-abilities. The means or tool used is the Web log, whose application yielded very satisfactory results. The objective of this article is to present a tool that has been very useful to us in this new normal. In conclusion, this project also allows the student to act as the main actor in their learning, being autonomous and independent in the search for their knowledge.
\end{abstract}

Keywords: nursing, digital platform, learning, blog, consultation.

${ }^{1}$ El término Web log significa blog o bitácora.
Recepción: 25/05/2021. Aprobación: 08/12/2021. Dol: http://doi.org/10.22201/cuaieed.16076079e.2022.23.1.7 
Licenciada en Enfermería, Maestra en Enfermería con Énfasis en Administración de Servicios de Enfermería, Doctora en Ciencias de la Salud en el Trabajo. Institución de adscripción: Facultad de Enfermería de la Universidad Veracruzana, región Poza Rica-Tuxpan, docente de tiempo completo categoría C, perfil PRODEP, productividad nivel VI. Integrante del núcleo del CA "Prácticas de Salud y Educación", LGAC "Salud y Educación", docente en enfermería y Licenciada en enfermería certificada, funciones sustantivas: docencia, gestión, tutoría, producción académica-científica (publicación en revistas arbitradas e indexadas en el ámbito nacional e internacional, libros y capítulos de libro en las áreas de salud, educación e investigación), ponencias a nivel nacional e internacional.

Fabiola Cruz Núñez

facruz@uv.mx orcid.org/0000-0002-0498-7645

Licenciada en Enfermería, Maestría en Ciencias de Enfermería, Doctora en Educación. Institución de adscripción: Facultad de Enfermería de la Universidad Veracruzana, región Poza Rica-Tuxpan, docente de tiempo completo categoría C, perfil PRODEP, productividad nivel VI. Líder del CA "Prácticas de Salud y Educación", LGAC "Salud y Educación", funciones sustantivas: docencia, gestión, tutoría, producción académica-científica (publicación en revistas arbitradas e indexadas en el ámbito nacional e internacional, libros y capítulos de libro en las áreas de salud, educación e investigación), ponencias a nivel nacional e internacional.

\section{Guadalupe Santes Saavedra}

gsantes@uv.mx orcid.org/0000-0001-5237-0570

Licenciada en Enfermería, Maestría en Ciencias de Enfermería, Doctora en Desarrollo Educativo. Institución de adscripción: Facultad de Enfermería de la Universidad Veracruzana, región Poza Rica-Tuxpan, docente de tiempo completo categoría B, perfil PRODEP, productividad nivel IV. Integrante del núcleo del CA "Prácticas de Salud y Educación", LGAC "Salud y Educación", docente en enfermería y licenciada en enfermería certificada, funciones sustantivas: docencia, gestión, tutoría, producción académica-científica (publicación en revistas arbitradas e indexadas en el ámbito nacional e internacional, libros y capítulos de libro en las áreas de salud, educación e investigación), ponencias a nivel nacional e internacional.

\section{Cynthia Lara González}

cylara@uv.mx

Licenciada en Enfermería, Maestra en Salud Pública. Institución de adscripción: Facultad de Enfermería de la Universidad Veracruzana, región Poza Rica-Tuxpan, profesor por horas-base, Enfermera General del Imss \#24.

Colaboradora del CA "Prácticas de Salud y Educación", LGAC "Salud y Educación", docente en enfermería y licenciada en enfermería certificada, funciones sustantivas: docencia, tutoría, producción académica-científica (publicación en revistas arbitradas e indexadas en el ámbito nacional e internacional, libros y capítulos de libro en las áreas de salud, educación e investigación), ponencias a nivel nacional e internacional. 


\section{Introducción}

E nuevo coronavirus ha afectado el empleo, la educación, la energía, la agricultura y otras áreas de la economía mundial, así como la salud emocional de los ciudadanos. Las Instituciones de Educación Superior (IES), incluidas las universidades, institutos universitarios y otros centros de educación terciaria, no son una excepción. Un artículo reciente apunta que esta crisis ha impedido que cerca de mil 300 millones de estudiantes de educación secundaria y terciaria en to do el mundo pudieran asistir a sus centros educativos, lo cual posiblemente tendrá un enorme impacto en la educación a nivel mundial (Naciones Unidas, s. f.).

Según las Naciones Unidas (s. f.), las IES pueden usar esta crisis como una oportunidad para el impacto académico, mediante la colaboración, como un mundo, una familia en estos tiempos de adversidad. Para superar esta crisis, éstas deben actuar rápidamente, pensar de forma innovadora, trabajar en conjunto para mitigar el impacto de este reto. Esto requiere un compromiso para cambiar nuestra manera de pensar sobre cómo planificar e implementar la educación, y usar soluciones novedosas para que su huella sea mayor.

Para ello, la educación superior se ha visto en la necesidad de modificar sus procesos de enseñanza-aprendizaje implementado nuevas estrategias, que, aunque algunas ya se utilizaban se hacían en menor proporción, pasando de una enseñanza presencial a una modalidad virtual o a distancia, buscando formas de dar continuidad al aprendizaje de los estudiantes tales como el e-learning o campus virtual (UNESCO-IESALC, 2020, p. 15).

El surgimiento de estos modelos formativos como el e-learning o b-learning (modalidad semipresencial), dan paso a que la tecnología sea fundamental en este nuevo contexto de enseñar y aprender (Global Campus Nebrija, 2016, p. 5). Aunque está situación para la mayoría de los estudiantes ha traído consigo una serie de complicaciones por la falta de equipo de cómputo o de acceso a internet sobre todo a la hora de la asistencia sincrónica a clases, es decir, en donde todos los participantes aprenden al mismo tiempo e interaccionan con los demás (Easy LMS, 2020).

Esto dio pauta para pensar en la creación de un espacio digital en el cual el estudiante pudiese encontrar a cualquier hora, en cualquier momento, información relacionada con las experiencias educativas o materias de Enfermería Farmacológica, Anatomía y Fisiología, y Práctica Profesional Comunitaria (Universidad Veracruzana, 2013, p. 127-128), por lo que, el objetivo de este artículo es presentar una herramienta que nos ha sido de gran utilidad en esta nueva normalidad.

Ante este contexto, es importante comentar que la virtualidad puede llegar a ser gratificante, productiva para ambos actores del proceso enseñanza- 
aprendizaje (estudiantes y maestros), siemprey cuando se efectúe de una manera adecuada, proactiva y receptiva; especialmente, los docentes universitarios son los convocados a participar en estas nuevas modalidades, poniendo en práctica la innovación, superación y adaptación a las necesidades del entorno educativo (Moreira-Segura y Delgadillo-Espinoza, 2015, p. 129).

Dentro de los fundamentos del aprendizaje en red se tiene al conectivismo y la teoría de la actividad, el primero se basa en que el conocimiento se difunde por medio de una red de conexiones, lo que implica que el aprendizaje radica en la capacidad de construir y traspasar esas redes, siendo el individuo un sujeto de aprendizaje, pero a la vez formando parte de éstas (Solórzano y García, 2016, p. 103, 105).

El principio del conectivismo es parecido a lo que se establece en el constructivismo social, en donde las interacciones de tipo social y cultural fungen como los engranajes de los mecanismos para el aprendizaje (Driscoll, 2005 citado en Solórzano y García, 2016, p. 105). La teoría de la actividad, como se comentó antes, también es un fundamento del aprendizaje en red, debido a que hace alusión a todo un sistema de actividad más allá de una persona, lo que permite guiar y acompañar al estudiante en su aprendizaje de manera flexible, y que se centra en la ejecución de tecnologías en la actividad del o los individuos, así como, en el uso y la exploración de los sistemas de información (Solórzano y García, 2016, p. 105).

\section{“Enfermería-Consulta”}

Se trata de un espacio digital que pretende dar respuesta a una emergencia profesional ocasionada por la COVID-19, y también a una tendencia en el uso de la Tecnología de la Información y la Comunicación (TIC). El objetivo principal de este espacio es brindar contenido que sirva al estudiante para su formación educativa, así como, el de ser un sitio para el intercambio de información profesional, que le ayude en el ser y hacer de la enfermería para proporcionar cuidado al individuo apegado en valores como el respeto, la responsabilidad sólo por mencionar algunos.

Enfermería-Consulta es una plataforma que sirve como recurso didáctico, permitiendo al estudiante acceder a sus aplicaciones, recursos y datos digitales de manera remota y desde cualquier dispositivo. En la plataforma, los estudiantes interactúan a través del chat o del envío de mensajes de esta manera adquiere dinamismo el proyecto, ya que el estudiante puede enviar sus comentarios, opiniones, dudas sobre el sitio o de la información que serán respondidas. El sitio es administrado y moderado por las creadoras y se actualiza cada cinco meses.

El espacio está estructurado por varias entradas con contenido relacionado a las experiencias educativas antes referidas, este responde a una necesidad 
detectada a través de la observación o por comentarios de los propios estudiantes, en donde los alumnos obtendrán, intercambiarán o reforzarán información para complementar el desarrollo de las experiencias educativas, y que además podrán consultar en tiempo diferido tal contenido. La plataforma se dio a conocer a los estudiantes que cursan tales experiencias educativas.

El medio instruccional utilizado es el Web log (blog), el cual constituye una herramienta de colaboración asincrónica (diferido en el tiempo) que origina la expresión de ideas, coloca contenidos a disposición de otras personas en la Web de forma clara y muy fácil de comprender. El blog, a su vez, permite una organización flexible e innovadora que facilita el diálogo de saberes, por lo que se beneficia una construcción colectiva del aprendizaje significativo al establecerse interacción entre los usuarios de una manera más crítica (Villalobos, 2015, p. 115).

Entre los recursos digitales manejados están: videos educativos, webs', e-books², diversos formatos (guía de valoración comunitaria, razonamiento diagnóstico, plan de cuidados, listas de cotejo, rúbricas, etc.), presentaciones, documentos portátiles o PDF, manuales, enlaces, productos de investigación, entre otros. El sitio es de reciente creación: se elaboró en octubre del 2020 y el hipervínculo de acceso es: http://consultaenfermeria.simplesite.com/.

En algunas de las entradas con las que se estructura Enfermería-Consulta se visualiza la página principal (ver figura 1) en donde se puede apreciar la bienvenida a los estudiantes, así como, el origen del espacio digital. En otra (ver figura 2) se hace alusión a la experiencia educativa de Enfermería Farmacológica en este espacio se encuentra información sobre aspectos teóricos de la farmacología, manejo de medicamentos, principio activo, clasificación y administración de medicamentos, etc.

Figura 1. Web log "EnfermeríaConsulta". Fuente: Elaboración propia.

${ }^{1}$ Webs, páginas electrónicas o digitales.

${ }^{2}$ El término e-books significa libros electrónicos o digitales.

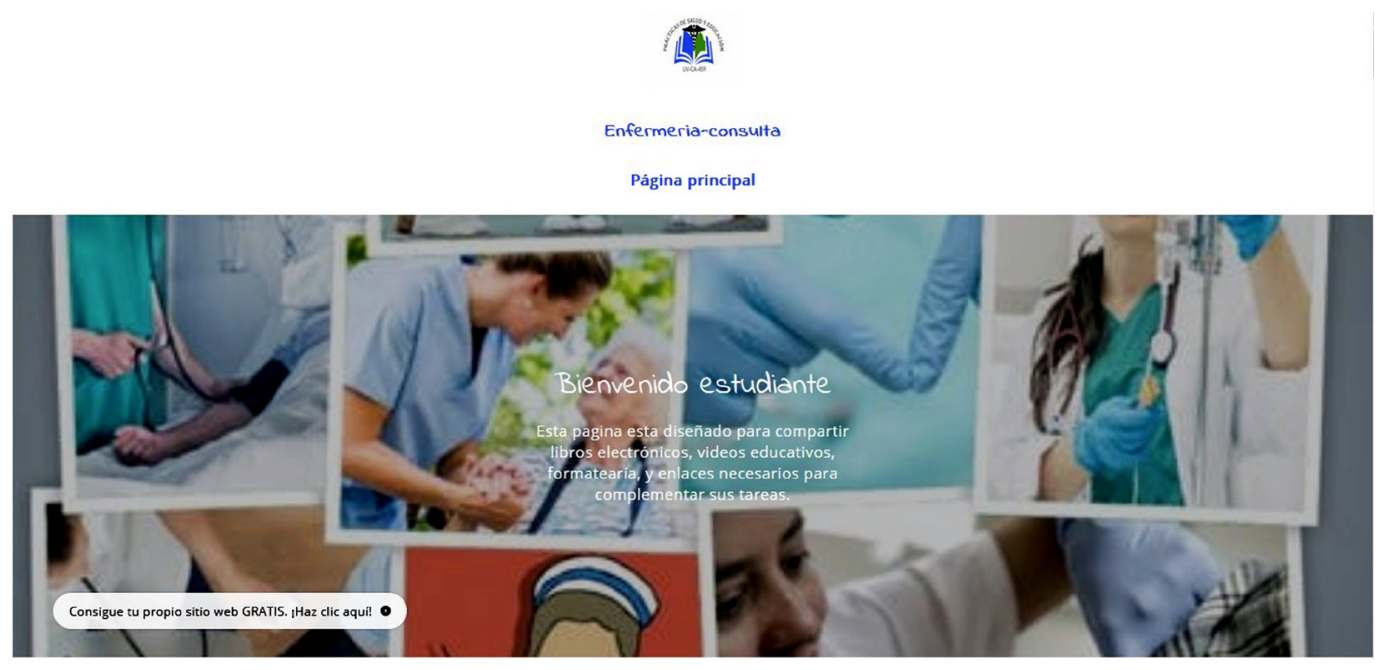


Figura 2. Ilustración del espacio de Enfermería Farmacológica. Fuente: Elaboración propia.

Figura 3. Entrada del Web log destinada a la formación en enfermería comunitaria. Fuente: Elaboración propia.

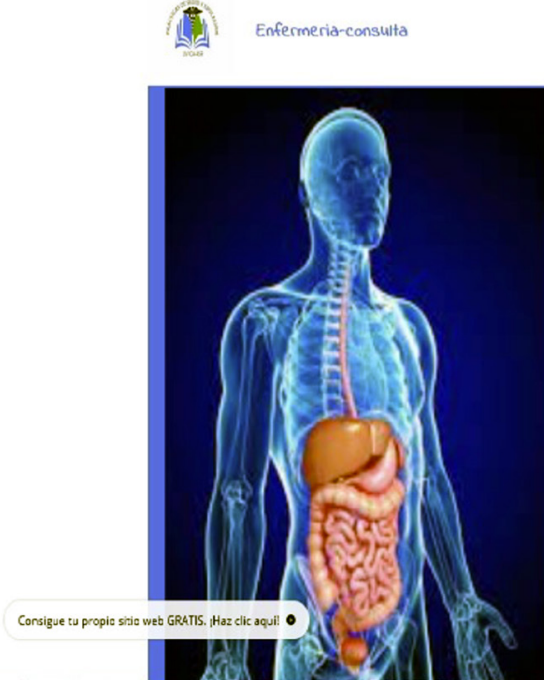

Página principal

\section{E.E. Anatomía}

Te ofrecemos videos educativos relacionados con

la anatomia y fisiologia humana, puedes

consultarlos al dar link:

Sistema muscular:

https://wwww.youtube.comiwatch?

https://wnww.youtube.comiwatchiv=kec10 1 rtww50

Sistema nervioso

https://wroww.youtube.comiwatchiveC4fmTtol bbo

https://www.youtube.com/watch?w=LASmiv8PeYM

https:/isurww. youtube.comiwatchiv=7r GUWCycSY

Pares craneales:

https:/vwww youtube.comiwatchiv=WSinTVOFawO

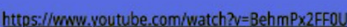

En relación con la experiencia educativa de Práctica Profesional Comunitaria, ésta permite al estudiante aplicar conocimientos teórico-prácticos focalizados en el fomento de una cultura de prevención y autocuidado en las personas vinculada con la atención primaria de salud (ver figura 3).
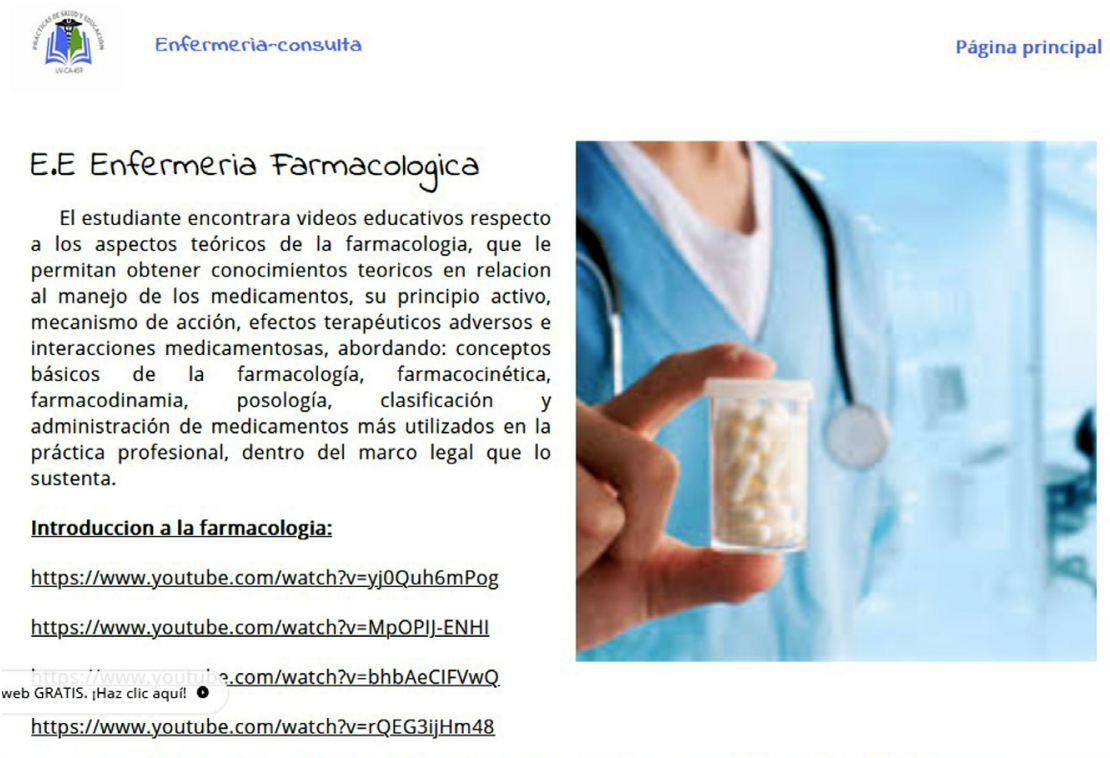

En otra parte (ver figura 4) se observa la plataforma para la experiencia educativa de Anatomía y Fisiología, cuya finalidad es brindar conocimientos sobre la estructura y funcionamiento de los distintos aparatos y sistemas que conforman el cuerpo humano.

La creación e implementación de este proyecto permitió obtener resultados que han sido muy satisfactorios, los estudiantes han referido: "el blog está bien estructurado", "me ha servido para complementar mis tareas", "puedo consultar 
Figura 4. Área de la experiencia educativa de Anatomía y Fisiología en EnfermeríaConsulta. Fuente: Elaboración propia. información diferente", "hay variedad en la información", "puedo consultar cuando yo quiera", "he reforzado mi aprendizaje con este sitio", "buena estrategias maestras", "gracias por la información", "maestras me parece que van por un buen camino, saludos y suerte con el sitio", "maestra gracias por disipar mi duda sobre la materia de enfermería farmacológica", "gracias por contestar a mi pregunta de la experiencia educativa de Anatomía", "me gusto el material de las materias", entre otros.

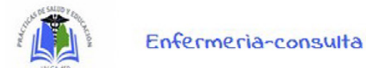

Página principal

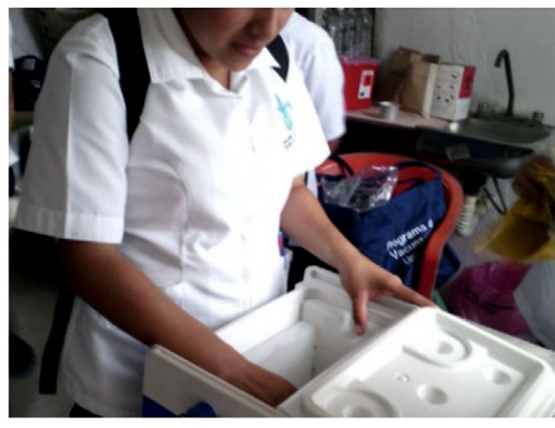

E.E de Practica Profesional

Comunitaria

Esta experiencia educativa, permite al estudiante aplicar conocimientos teórico prácticos focalizados en el fomento de una cultura de prevención y autocuidado en las personas usuarias de instituciones de primer nivel de atención y en la comunidad, en este contexto el estudiante de enfermería integra en su práctica profesional comunitaria los saberes de la atención primaria de salud, técnicas de abordaje comunitario y métodos estadísticos básicos.

b GRATIS. Haz clic aqui! $\bullet$

En este apartado encontraras videos educativos que te proporcionaran un gran apoyo para tu formacion en enfermeria comunitaria.

Hasta la fecha Enfermería-Consulta ha tenido 250 visitantes. A cualquier usuario se le pide que responda a cuatro preguntas que evalúan la calidad del sitio (ver más detalles en la tabla 1).

\section{Conclusiones}

El sitio digital ha impactado en la continuidad de los aprendizajes de los estudiantes, manteniendo una relación con lo que se especifica en el perfil de egreso, por ejemplo, contribuye en la adquisición de saberes teóricoscientíficos, enfocados a brindar el cuidado en la persona, familia y comunidad en las diferentes etapas de la vida, cuyo énfasis se enfoca en la promoción y prevención.

Consideramos que los resultados han sido satisfactorios. Casi la totalidad de los participantes ha comentado que la información de consulta y la aportación en su aprendizaje es buena, así como la retroalimentación recibida. Sin embargo, es importante mencionar que algunos estudiantes refirieron: "hace falta más contenido", "dar mayor difusión", "mejorar el tiempo de respuesta", etc., ante lo expuesto nos hemos planteado los siguientes elementos para mejorar el proyecto: 
- Mejorar el acercamiento con los usuarios y el posicionamiento, utilizando el chat.

- Crear una manera más fácil de encontrar el blog, a través de la difusión por ejemplo las redes sociales.

- Hacer lo posible por responder en el menor tiempo posible, estableciendo un periodo.

- Es indispensable difundir que los visitantes se suscriban a los comentarios.

- Para hacer más dinámica y atractiva la plataforma se pretenden premiar los comentarios más activos, por medio de un e-mail de agradecimiento.

\section{Referencias}

* Driscoll, Marcy (2005): Psychology of Learning for Instruction, Pearson PLC, Toronto.

- Easy LMS. (2020). Aprendizaje sincrónico vs. asincrónico: cuál es la diferencia. https:// cutt.ly/DUegsp5

* Global Campus Nebrija. (2016). Metodología de enseñanza y para el aprendizaje. 1-136. https://cutt.ly/VUeggWz

* Moreira-Segura, C. y Delgadillo-Espinoza, B. (2015). La virtualidad en los procesos educativos: reflexiones teóricas sobre su implementación. Tecnología en Marcha, 28(1), 121-129. https://cutt.ly/XUeqvLp

* Naciones Unidas. (s. f.). COVID-19 y educación superior: aprender a desaprender para crear una educación para el futuro. https://cutt.ly/6UeqWsk

* Solórzano, M. F. y García, M. A. (2016). Fundamentos del aprendizaje en red desde el conectivismo y la teoría de la actividad. Revista Cubana de Educación Superior, (3), 98-112. http://scielo.sld.cu/pdf/rces/v35n3/rces08316.pdf

* unesco-Iesalc. (2020, 6 de abril). Informe COVID-19 y Educación Superior: de los efectos inmediatos al día después. Análisis de impactos, respuestas políticas y recomendaciones. https://cutt.ly/jUeqTTY

* Universidad Veracruzana. (2013). Plan de Estudios 2013. Licenciatura en Enfermería. https://www.uv.mx/pozarica/enfermeria/files/2016/06/Plan-de-Estudios-2013. pdf

* Villalobos, F. E. J. (2015). Uso del Blog educativo en procesos de aprendizaje de Educación Ambiental. Revista de Investigación, 39(85), 115-137. https://www. redalyc.org/pdf/3761/376143541007.pdf 


\section{Apéndices}

Tabla 1. Instrumento de evaluación y resultados cuantitativos.

Fuente: Elaboración propia.

\begin{tabular}{|l|c|c|c|}
\hline \multicolumn{1}{|c|}{ Ítems } & Calificación & $f$ & $\%$ \\
\hline 1. ¿Qué calificación le das al sitio del 1 al 10? & 9 & 62 & 25 \\
& 8 & 113 & 45 \\
& 7 & 35 & 14 \\
& 6 & 40 & 16 \\
\hline 2. ¿Del 1 al 10 como calificas tú experiencia en la & 10 & 69 & 28 \\
página web? & 9 & 89 & 36 \\
& 8 & 81 & 32 \\
\hline 3. ¿Consideras que es buena, regular o deficiente la & 6 & 11 & 4 \\
\hline información encontrada en este espacio? & Buena & 198 & 79 \\
& Regular & 43 & 17 \\
\hline 4. ¿Te ha ayudado está plataforma en tu educación, & Deficiente & 9 & 4 \\
\hline responde sí o no? & Si & 244 & 98 \\
\hline Total & No & 6 & 2 \\
\hline
\end{tabular}

\section{Cómo CITAR ESTE ARTículo}

Lavoignet Acosta, Blanca Judith, Cruz Núñez, Fabiola, Santes Saavedra, Guadalupe y Lara González, Cynthia. (2022, enero-febrero). Enfermería-Consulta: una plataforma digital que apoya el aprendizaje. Revista Digital Universitaria (RDU), 23(1). http://doi.org/10.22201/cuaieed.16076079e.2022.23.1.7 